\title{
A Proposal for The Development of Sport at Abdelmalek Essaâdi University
}

\author{
Soulaiman HARFOUF \\ Interdisciplinary Research Laboratory on Pedagogical Engineering \\ Higher School of Education-Abdelmalek Essaâdi University (UAE), Tetouan, Morocco
}

\begin{abstract}
This study deals with sport at the Abdelmalek Essaâdi University-Tetouan Morocco (UAE). It adopts a problematic related to the development of the sport/Physical Education (PE) system in this Moroccan public university. After identifying the factors responsible for the dysfunction of this system [1], the study makes a proposal to revitalise Sports Activities and PE in the KAU and its affiliated institutions. In order to answer the question of effective measures to be taken to improve the quality and quantity of the PES/sport system in the KAU, our method of approach consists of intervening by positivising, in the particular context of the UAE, the negative indicators. Upstream, we propose an operational approach with the aim of bringing together the favourable conditions for the success of this initiative.
\end{abstract}

Keywords: University Sport (US) - Physical Education (PE) - Development - Abdelmalek Essaâdi University (UAE).

\section{INTRODUCTION}

University Sport (US) in Morocco is increasingly marginalised [2]. It is confronted with a number of dysfunctions that limit its contribution to the development of national sport and, beyond that, its participation in the social and human development process of the student population [3].

Indeed, since the integration of sport into university programmes and activities following the creation of the first Moroccan university in 1959, university sport has been very popular. Not only did it meet the expectations of all its actors, but it also constituted a melting pot of elite and mass practice occupying, with school sport, a place of choice in the development of sport in Morocco [4].

Today, despite the increase in the number of students enrolled in higher education, the US seems to have ceased to play this leading role and is struggling to fulfil its missions. In other words, the US seems to be in crisis at several levels: lack of qualified human, financial, material and infrastructural resources, managerial dysfunctions and lack of governance [5].

Sport and physical education in UAE schools are no exception. Ad hoc, sectoral and localised initiatives have been attempted without producing the desired effect [6].

Other more operational attempts preceded it, but without being approached in a comprehensive and systemic way and without maintaining the pace and intensity of the reform effort initially undertaken [7].
Consequently, and after having identified, in a previous study [8], the sources of dysfunction of the US/PE system in the specific context of the UAE, this study proposes a global and operational approach to the development of sport and PE in the UAE, focused more on the practice of good governance and modern managerial principles in order to "guarantee" the success of this project.

\section{Problematics}

It has been established that the Sport/PE system in the UAE suffers from inadequacies and deficiencies in terms of infrastructure, facilities, supervision and governance. The problem of the development of this system requires the positvisation of its negative indicators taking into account the elements of the particular context of the UAE.

We therefore formulate the following question: What approach should be developed and what measures should be taken in order to guarantee a tangible and sustainable development of the PES/sport system in the UAE?

\section{Methodology}

Our method of approach is first to seek the support of the UAE leadership by advocating a rigorous approach and efficient organization. The UAE would then proceed to build capacity at all levels. The next step is to intervene rationally for change by collectively defining the expected results, the strategies to be followed and the responsibilities of each project actor. A strategy for 
motivating and raising the awareness of the actors, the establishment of a culture of communication and coordination, the continuous evaluation of the actions undertaken and the opening up to the external environment of the KAU would constitute guarantees of the success of this project.

\section{Proposal}

\section{A. Seeking membership of the UAE}

\section{1) From the approach first}

We are looking for the University to have the ambition to upgrade and develop PES, sports leadership and competition within the UAE. However, the project can only germinate if the main actors share the diagnosis and the objectives to be achieved.

Our approach aims first of all to convince the President of the UAE and the heads of the various establishments belonging to the UAE and the two university residences (decision-making bodies) of the importance of this project.

The following arguments should be put forward:

- The legal argument: in favour of effective, supervised, democratic, generalised and sanctioned university sport in training programmes, particularly in institutions with regulated access;

- The economic argument: The implementation of the project and its operationalisation will only require a very reasonable budget, given that this project is oriented more towards the optimisation of existing resources and the creation of additional resources through partnership projects, notably with the local authorities and public and private sports organisations in the region.

- Moreover, this project can be economically profitable in the long and medium term. University sport will potentially generate funds through sponsorship partnerships in addition to government and local authority grants. The commercial sector will be able to use university sport as a means of advertising.

- The scientific research argument: Role and mission of the university in the production of scientific knowledge in the field of science and technology of physical and sports activities (STAPS). On this point, a structure is already in place at the Higher School of Education (HSE), belonging to UAE. It is the Sports Management and Administration (SMA) programme. We propose that the HSE will lead the implementation of this project.
- The pedagogical argument: the importance of PE and US in the overall training and intellectual and professional performance of the student.

\section{2) Getting organised}

Although conceived according to hierarchical levels of responsibility, the proposed organization does not fall within the Taylorian or sequential model of project management, in which the implementation passes from one profession to another (the R\&D department designs the product, then passes on its work to the production department for operational implementation, which in turn passes on the work to the sales department, which ensures the commercial launch of the product [9]. This type of development, described as 'sequential', because a stage can only be started if the one before it is completed, is not appropriate for our proposal).

We opt instead for the concurrent engineering model or concurrent approach [10], illustrated by the metaphor of the rugby team, in which each member of the team progresses at the same time as the others, while many combinations remain possible at almost all stages of the game [11].

In this concurrent engineering model, all the trades in the organization work simultaneously on the project and therefore interact continuously. This organizational mode consists of involving all the players in the project in an iterative and interactive way. Each member of staff puts his or her skills at the service of the project. Defined in this way, the project moves towards a matrix organization by project.

In the case of the UAE Sport Development Project, a vertical structure (the three strategic, organizational and functional levels) is combined with a horizontal structure of commissions, committees and teams. At this level, the principles of functional reporting and hierarchy intersect (Fig.1). The points of intersection are the positions. The advantages over a functional organization are as follows:

- Individuals can be chosen according to the needs of the project ;

- The use of a project team that is dynamic and able to detect problems early and even anticipate them ;

- $\quad$ Project managers are directly responsible for the completion of the project, within a specific timeframe and budget;

- The use of a project team that is dynamic and able to detect and anticipate problems at an early stageles. 


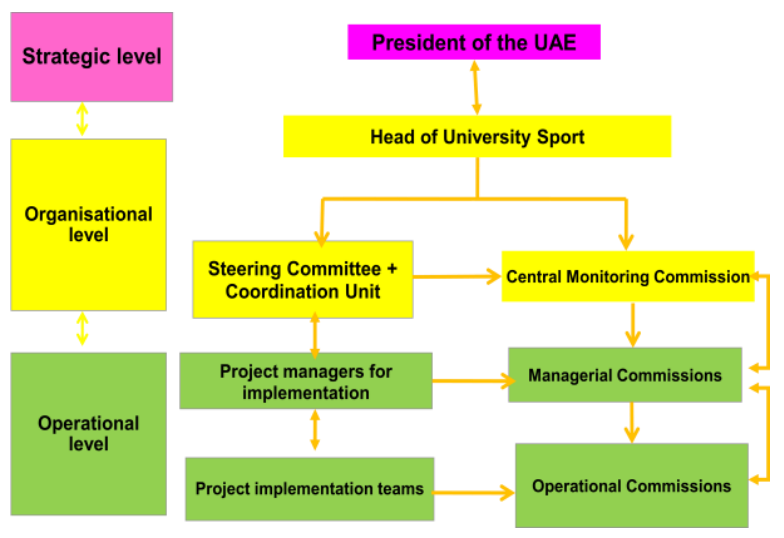

Figure 1. Proposed human organization.

\section{3) The context and advantages of the UAE}

It is then a question of highlighting the advantages that the UAE enjoys in its context.

Indeed, the geographical location of Abdelmalek Essaâdi University gives it a unique and incomparable advantage: in addition to the cities of Martil, Larache, Al Hoceima, Chefchaouen and Ksar El Kébir, the University spreads over the two major cities of the region, Tangiers and Tetouan. It is located on a site that is home to many riches. It contains:

- Large number of students and researchers;

- Home to prestigious sports clubs ;

- The only HSE in the region that offers a professional training course in sports management and administration, the only one in the region ;

- Numerous institutes and schools for specialized training ;

- Private companies...

The question that arises therefore is how to mobilise all this capital, this enormous potential of human and material resources?

\section{4) From the shared diagnosis}

It is a question of sharing the conclusions of the diagnostic effort of the current state of the sport/EPS system in the UAE. In this sense, it is now easy to agree that sport in the UAE-Tetouan suffers from insufficiency and deficiency in terms of:

- Infrastructure ;

- Equipements ;

- Management ;

- Governance.

The results presented in a previous study [12] attest to this. In practical terms, the aim is to design a schematic, summarised and precise presentation of the main sources of dysfunction in the Sport/PE system at the UAE and to present it to the President of the University and in the presence of the heads of the various institutions under its authority, as well as other people concerned. We will choose the PowerPoint application as a presentation medium to carry out this action. The objective of this preparation is above all to qualify the nature of the project to be carried out on the basis of its objective complexities (Fig. 2). The cartographic representation of the project will also enable the decision-makers and the project leader to better visualise the strategies and actions to be adopted.

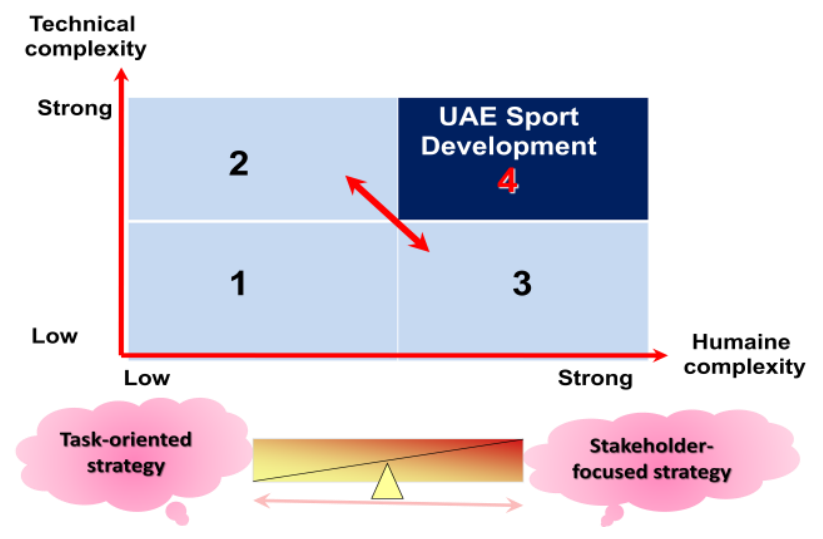

Figure 2. Project Mapping (UAE Sport Development Profile).

The diagram shows that the UAE sport development project has to deal with two types of complexity. The first is a human complexity related to the actors of the system as described above.

The second complexity relates to the technical factor concerning logistics (infrastructure and equipment) and financial means.

The entry is therefore twofold. On the one hand, it is a question of getting the various stakeholders to accept the change, in particular the project management team (sports service managers, supervisors, team of project leaders) and the beneficiaries (students).

In order to achieve this, it is useful to anticipate the uncertain reactions of these actors and to adapt the interventions to their attitudes towards the planned change (Fig.3).

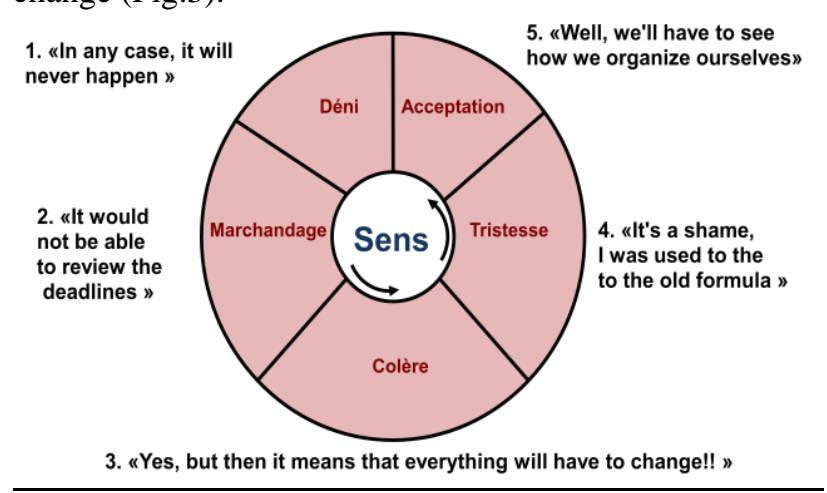

Figure 3. Typical attitudes to change. 
On the other hand, it is also necessary to mobilise the necessary budget for the upgrading of the sports infrastructure and the purchase of sports equipment. For both types of intervention, two prerequisites are essential in our approach (Fig.4):

a. To transform management methods by adopting a project-based organization on the one hand, and

b. Implementing a steering and implementation system for the programme by setting up a battery of monitoring tools and indicators. [13]

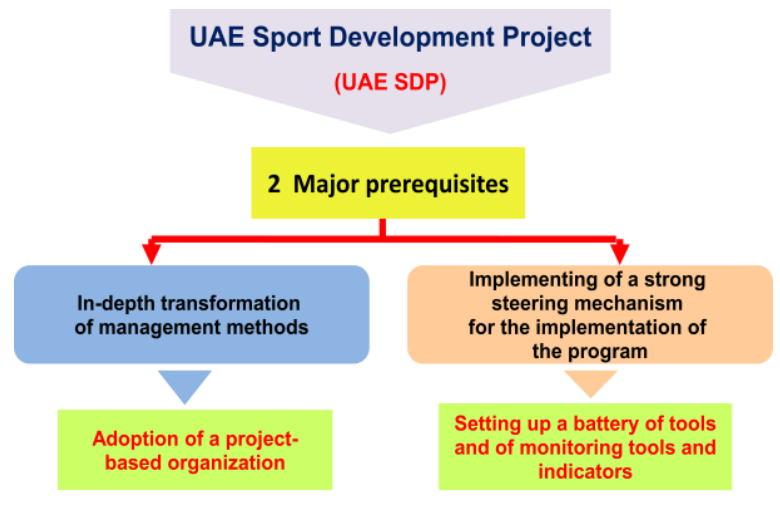

Figure 4. Prerequisites for successful completion of the UAE Sport Development Project.

5) Objectives to be achieved and development of a shared design

Une A second question is also decisive: What is the ambition of Abdelmalek Essaâdi University and the ENS in terms of promoting university sport?

If the agreement is reached on the subject of the observation, the objectives to be reached seem obvious: to act on these main aspects: infrastructure, equipment, supervision and governance.

In short, the aim is to improve the negative indicators, both qualitatively and quantitatively. However, the restructuring and upgrading of the functioning of sport at the UAE should in principle lead to the achievement of certain goals, namely the progressive generalization of access to university sports services for the maximum number of UAE students on the one hand, and the restructuring of elite sport to enable talented students to excel in their sports specialities and to represent their university with dignity in competitions organized at national, regional and international level on the other.
The UAE's adherence to these two major objectives would be a pledge for the next steps.

These objectives will help to orient and give meaning to the actions undertaken within the framework of the project (Fig.5). Indeed, meaning comes first in the process, which comes first in the content; meaning comes first in the process, which comes first in the content. The project must be driven by the meaning we want to give it. The work processes only follow on from this. Very often, the hierarchy is reversed: the focus is on the deliverables, to the detriment of the processes and even more so of the meaning. This leads to the paradoxical situation of projects that are impeccably executed in terms of content, but which do not generate support.

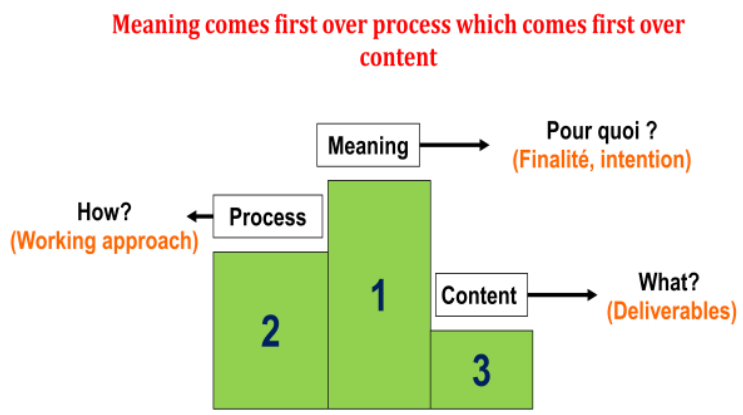

Figure 5. Predominance of meaning over pair Process-content in the UAE SDP

The meaning takes precedence over the process, which takes precedence over the content. The project must be driven by the meaning it is intended to give. The work processes only follow from this. Very often, the hierarchy is reversed: the focus is on the deliverables, to the detriment of the processes and even more so of the meaning. This leads to the paradoxical situation of projects that are impeccably executed in terms of content, but which do not generate support.

In our case, the UAE-SDP needs to get the actors on board. It is recommended that they understand the need for change and become ready to commit to it without the risk of backtracking. We consider that an input focused on the meaning of the action in the eyes of the actors would increase the chances of success of this project.

\section{6) Methodological approach}

We illustrate the methodological approach in the figure below (Fig. 6): 


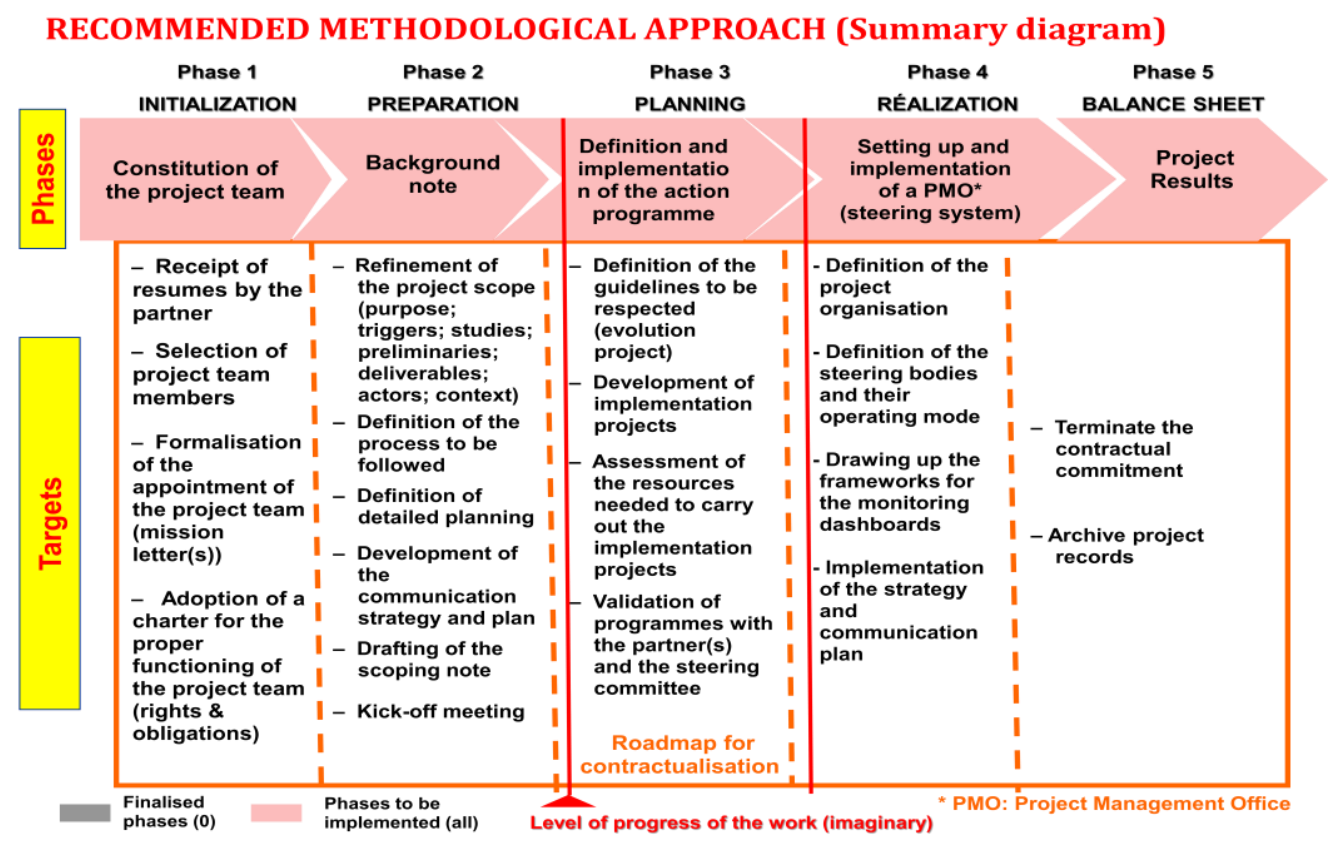

Figure 6. Methodological approach.

\section{B. Acting for change}

\section{1) The implementation and remit of the UAE}

Implementation must be preceded by the definition of operational objectives on the one hand and the availability of human resources, material resources and efficient, appropriate and effective tools on the other.

The Abdelmalek Essaâdi University has the ambition to open up to its environment and take up the challenge of developing an integrated vision of sport and physical activities: the idea of the CLAPS (Leisure and Sports Activities Center [14]. It aims to:

- Promote a wide range of sports activities and combine sports and leisure activities with physical education;

- Develop collaboration, cooperation and interweaving between school, university and civilian sport ;

- Combine elitist and mass practice ;

- Initiate research and experimentation projects ;

- Provide targeted training in sport and physical education ;

- Provide targeted training for specific needs (private halls, clubs, federations....) ;

- Initiate actions in partnership with public and private entities, national or external...
The mobilisation of decision-makers and public opinion (debate on the crisis in sport, unsatisfactory results at the Olympics, national conferences on sport, royal letter, etc.) can be used to the advantage of this ambition...

How can this be done?

It should be noted that this vision is not the result of an abstract construction, nor does it derive from a desire and overflowing enthusiasm. It is the fruit of in-depth reflection, fed with information and data from the reality of the context and based on an objective and rigorous analysis.

The design must be translated into concrete terms by drawing up operational proposals which would constitute both the choices to be made and the directions to be followed. (Cf. Methodological approach).

\section{2) The scope of the UAE's intervention in the field of university sport}

It is expected that those in charge of Abdelmalek Essaâdi University will become resolutely committed to a whole programme of activities which, while innovative, seeks to reinforce and consolidate the achievements. By adopting a participatory approach, they tend to involve the various stakeholders in the development and implementation of the programmed actions.

The involvement of the HSE in the revitalisation of university sport as a central actor. This institution is both an incubation centre for skills and a testing and experimentation laboratory. 
The launch of a pilot project of cooperation between school and university sports which will result in specific training cycles in sports disciplines at the HSE and the launch of a university and school championship in different sports.

The creation of a structure specifically in charge of the management of university sports activities directly attached to the Presidency.

The upgrading of the infrastructure and equipment of the Higher School of Education. Prospecting for the creation of a CLAPS.

\section{3) Providing the UAE with a minimum structure for US intervention}

The typical pattern of the structure and organization of the US at the UAE level follows the same principle of the national organization; it is divided into two levels. The base is formed by the academic institutions, which include the public faculties, schools and higher institutes and the two large university halls of residence. These are responsible for managing sports activities internally, locally, and it is up to them to guarantee the participation of their selections in inter-institutional sports competitions (providing the human and material resources). It is also up to them to organize themselves to make physical education a compulsory part of the training curricula, depending on the resources available;

The second level constitutes the field of intervention of the Abdelmalek Essaâdi University, notably by:

- The supervision and management of inter-school competitions

- The preparation of the university's representative teams for the national championship and the management of any initiative aimed at promoting sport on the university site.

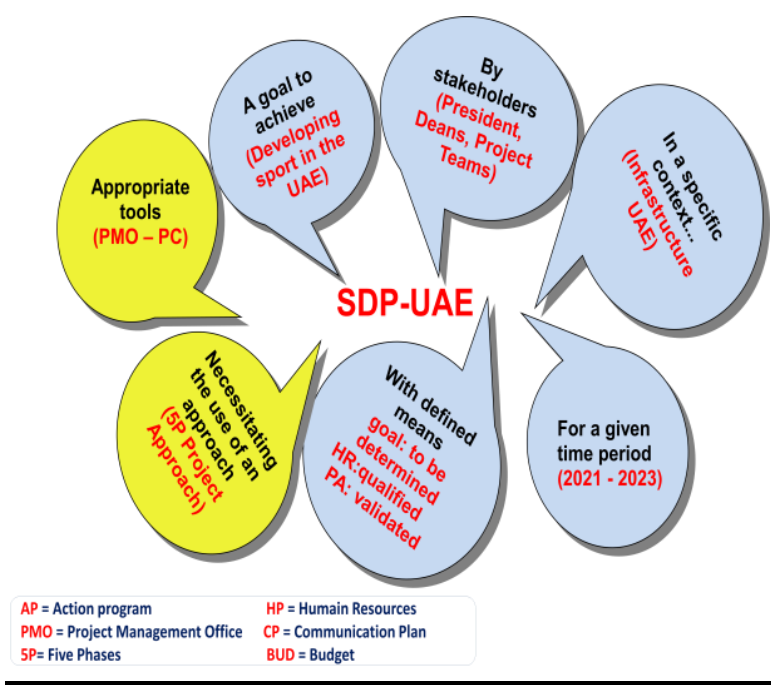

Figure 7. Operational definition of the UAE-SDP.
5) The sports office

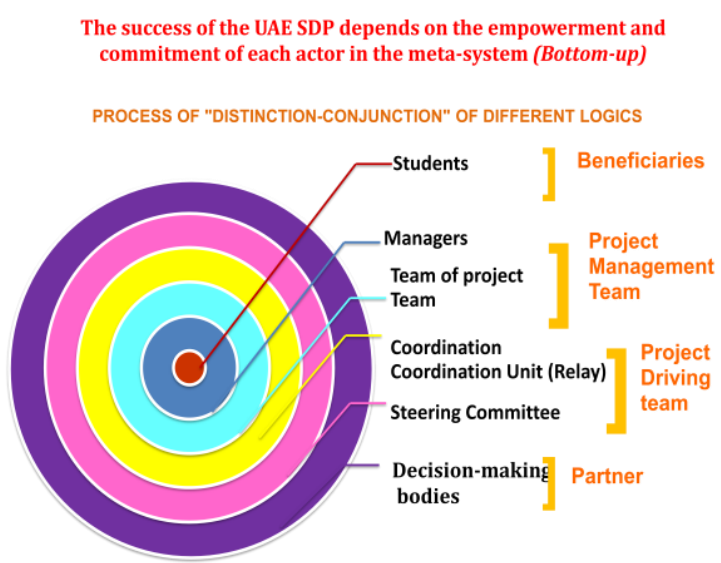

Figure 8. Process of "distinction-conjunction" of different logics.

This is a starting structure around which to build in a pragmatic and methodical way.

a. Composition:

The focus being initially on team sports and athletics. It is recommended to designate:

- One person responsible for each speciality (VB, FB, BB, HB, Rugby);

- One person responsible for athletics and individual sports;

- A leader for women's sport;

- An administrative manager;

- A permanent secretariat.

b. Constitution of the project team:

Receipt of CVs by the sponsor;

- $\quad$ Selection of the project team members;

- Formalisation of the appointment of the project team (mission letter(s));

- Adoption of a charter for the proper functioning of the project team (rights \& obligations).

c. Affectation :

Higher School of Education (but this presupposes that the infrastructure and equipment are upgraded and that special staff are assigned to maintain the premises...).

In addition, the group must have:

- a meeting room ;

- $\quad$ filing and storage facilities ;

- communication facilities.

d. Attributions: (Fig. 9)

They are managers responsible for the promotion, development, supervision, organization and animation of sports activities by speciality.

The main duties of the sports managers are: 
- To establish the calendar of inter-school matches by discipline;

- To proceed to the programming and the homologation of the matches;

- To ensure the coordination between the different persons in charge of the discipline, in the different university establishments, particularly for the establishment of the licenses of the selections, the arrangement of the training schedules and the designation of referees;

- To prospect and identify the best elements for the constitution of the UAE selections;

- To ensure jointly with the technical supervisors on the preparation, the supervision and the management of the selections of the Abdelmalek Essaâdi University, in the regional and national competitions in the various disciplines;

- To establish a circumstantial report on the main events;

- To draw up an annual evaluation report which gives an account of the tasks accomplished and to suggest the proposals which are necessary for the future.

e. Profile of the leader person and tasks to be undertaken in the immediate future:

He is a facilitator who has the strength of character and competence. He or she must be sufficiently informed about the conditions in which the discipline is practised at the various levels and equipped to make the most of the context. The discipline leader must be essentially a leader of men.
In the immediate future, the leaders have to carry out four main tasks simultaneously:

- To prepare, in collaboration with the team in place, the conditions for the launch of the 20132014 university meetings, planned in principle for November ;

- To proceed with the final approval of the meeting calendars; To ensure the conditions of implementation of these calendars ;

- To proceed to the prospection and identification of the best elements likely to integrate the selections of the University ;

- To establish, within a maximum of 45 days, a report on the state of the discipline, focusing on the infrastructure, equipment, supervision and operating procedures.

- To ensure the coordination and follow-up of actions, a monthly meeting could be organized at first; the spacing of these meetings could be done afterwards according to the needs.

\section{f. Recruitment conditions}

Recruitment must be based on the formalities and procedures in force. These must be fully known to the managers. If necessary, individual contracts should be drawn up.

NB: Since the material motivation is not very substantial, it is recommended that the conditions of recruitment be specified in such a way as to assess the determination of the commitments from the outset. There are choices to be made, and no one is forced to make a commitment without conviction. [15]

\section{Roles and responsibilities in the organization chart}

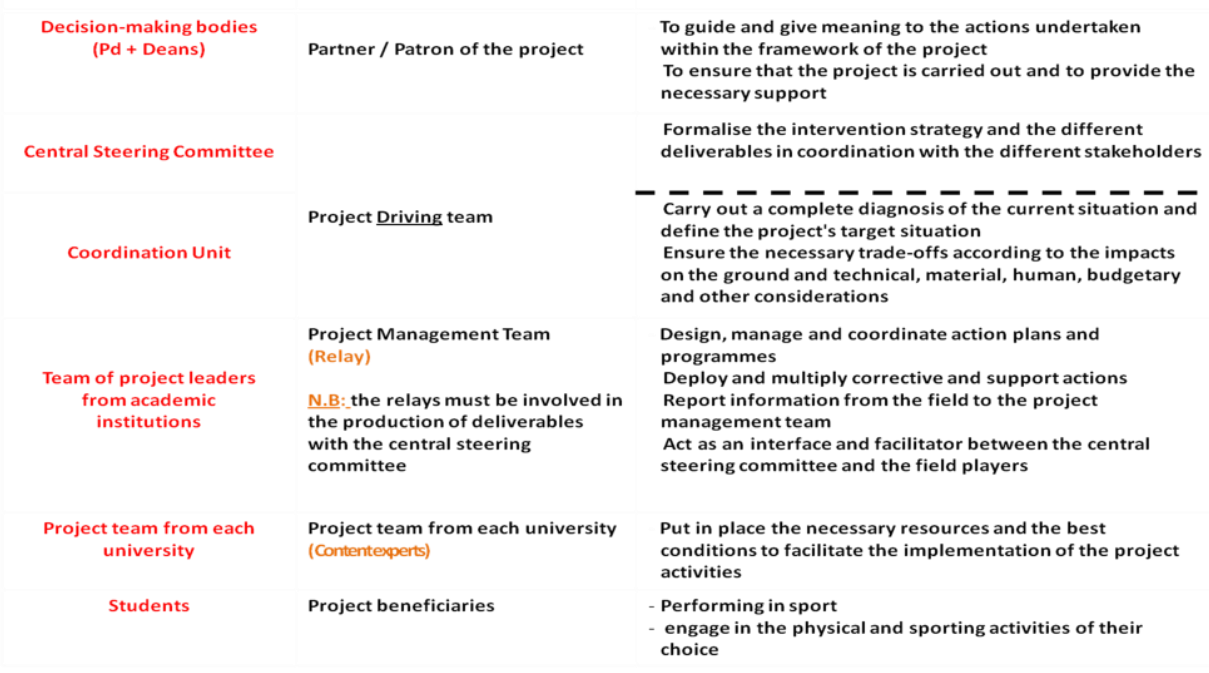

Figure 9. Roles and responsibilities in the organization chart. 


\section{Towards a progressive programming of sport and $P E$ in academic institutions}

The development of sport at the UAE is also materialised by the introduction of $\mathrm{PE}$ in the training programmes of university students [16]. Becoming compulsory, the physical education and sport sessions would be democratised on the whole population of the UAE-Tetouan students. Physical activities and sports will be imposed in the curricula and the raison d'être of PE will then be scientifically and culturally justified.

\section{1) Gradually make it compulsory in students' timetables}

Attempting to make PE compulsory in students' timetables requires the redefinition of the orientations assigned to university sport. The latter, in view of all the above-mentioned difficulties, seems to have failed in its missions. These difficulties encountered by this sector of sports practice have shown that the institutional structure of the Royal Moroccan Federation of University Sport and its regional leagues do not fully ensure the missions assigned to them.

The aim seems to be to increase the number of members and to massify the practice of sport while raising its level in educational institutions. In fact, we suggest that sport be made progressively compulsory for all students in DUT (preparing the University Diploma of Technology), in preparatory classes integrated into engineering schools and in the Bachelors cycle, at the rate of one module per month per cycle of the training course.

In order to revalorise physical and sports activities at university, to adapt them to the needs of students, and to give sport a real place in university education, it is judicious to require, for any new application for accreditation or for the renewal of a course, that at least one module dedicated to sports activities be included in the syllabus and subjects of each course, regardless of the speciality. While open access institutions will find it difficult to operationalise this measure, university schools and institutes can begin the work, given the small number of students, the availability of sports facilities and the possibilities of supervision by officials or temporary staff.

\section{2) Capacity building at all levels}

The UAE should commit itself to providing its EUs with infrastructure, material, financial and human resources, for a better functioning and a good influence of sports activities by allowing local sports structures (sports offices, university sports associations, sports clubs...) to fulfil their missions efficiently.

a. Sports infrastructure

The aim is to develop modern sports infrastructure and to provide university sport with the facilities it needs. The
UAE is called upon to develop a strategy for the redevelopment and construction of new sports facilities in order to provide each university with an appropriate infrastructure.

While waiting for the realisation of these redevelopment and construction projects, and with a view to sharing the use of public school and university facilities in the vicinity, it is becoming urgent to think about facilitating access to existing infrastructures for students of institutions that do not have them. It is also appropriate to enter into strategic partnerships with the Regional Academy of Education and Training (AREF) Tangier-Tetouan-Al Hoceima and its various provincial directorates in order to exploit the sports facilities available in the high schools and colleges of the region's cities. Recourse to the lease of private and public sports halls can be envisaged in certain cases [17].

The construction, refurbishment and mobilisation of all existing sports facilities aims at improving the conditions of practice, in particular through the enlargement of quality sports areas, fitted out and secured so as to allow the students of the UAE to evolve in sports under plausible conditions.

To facilitate the use of these facilities by students, a "sports office" will be created in each university, a onestop shop that aims, among other things, to simplify the procedures for students by giving them immediate access to the variety of sports facilities on offer, and to get the various actors in the field of sport at the university to work together.

b. Financial resources

As far as financial resources are concerned, university sport managers and decision-makers are invited to put this sector at the centre of their concerns and to allocate the budget that will ensure its smooth running. University sport will generate funds through sponsorship partnerships in addition to subsidies from public authorities and local communities. The commercial sector will be able to use university sport for advertising.

It is necessary to think about setting up a marketing department capable of marketing the brand image of Moroccan university sport, which will make it possible to generate funds through advertising displays at university sports events. The establishment of a marketing department at the level of the UAE-Tetouan regional league will also allow us to look for more sponsors and patrons both for the events organized and for the athletes excelling in certain disciplines. In this sense, we encourage the pilot initiative set up by the "Management and Administration of Sport" department and the Moghreb Atletico Tetouan Club to promote university sport on the training and expertise exchange side. We recommend that this partnership be strengthened and that others be established. In this sense, the UAE is called 
upon to open up more to its economic environment and become more permeable to initiatives with an economic stake in the socio-educational context.

Furthermore, we aim to introduce a compulsory "sports fee", the amount of which would be set by the University (paid at the same time as the registration fees) in order to contribute to the financing of the maintenance of sports facilities and to ensure their durability and functionality.

\section{c. Human resources}

In terms of qualified human resources, we recommend that all UAE universities recruit sports supervisors. The UAE will mobilise the motivated PE teachers of the AREF Tangier-Tetouan-Al Hoceima and the sports managers of the youth and sports delegation on the one hand, and will ensure the training of university managers and instructors on the other hand; in fact, the University has entrusted the coordinator of the "Sports Management and Administration" course and his pedagogical team with the task of drawing up a harmonised training plan for temporary teachers and those in charge of university sports at the UAE and the various institutions under its authority.

University sport suffers from a great lack of permanent and sustainable sports supervision. The recruitment of full-time coaches can improve the practice of sports at the UAE. The university league and the presidents of the different institutions of the UAE must mobilise in this sense.

\section{3) A new strategy to motivate and raise awareness among stakeholders}

It is necessary to think about offering more advantages to sports students, especially elite ones, in the sense of facilitating their practice of university sports at the same time as the normal pursuit of their studies. This means, for example, providing them with the means and conditions for academic success (reinforcement and remedial courses, bonus points for students who are champions or winners of university sports games. These same students will also benefit from additional timetables compared to others as well as scholarships. It will also be necessary to provide them with accommodation and to take into account the university requirements in relation to the imperatives of university sport...).

The creation of privileged conditions for high-level sportsmen and women at university would also ensure that high-level sportsmen and women can study in conditions that allow them to practice their sport and/or resume their studies after their sports career.

\section{4) Practising good sport governance}

Good governance for sport development in the UAE includes effective organization, clarity and communication of objectives, and the constant use of evaluation.

\section{a. Collectively developing a strategy}

The UAE-Tetouan is responsible for the management of the "university sport" dossier within the limits of its competences. Therefore, institutional actors (all UAE constituents) must contribute to the definition of a strategic vision, the mobilisation of resources and competences and the continuous evaluation of performance.

Indeed, the leaders of the UAE are called upon to agree and identify with a clear vision, mission and strategic directions for the development of the sport/physical education system. These orientations must be congruent with the recommendations of the national charter of education and training, with the provisions of the law 30-09, with the project $n^{\circ} 6$ of the plan of the Ministry of Higher Education, Scientific Research and Executive Training, 2013-2016, with the salient features of the 2020 vision of Moroccan sport such as "To anchor the practice of sport and its values in the daily life of each one" and "To make Morocco a land of sport and a crucible of champions".

Without confusing vision, mission and objectives, those in charge should not be satisfied with the operational register and the day-to-day management of sport at the UAE. The overall strategic framework will take the form of an evolutionary project. The latter will give meaning to the implementation projects (subprojects which in turn contribute to the construction of the evolution project) according to the following scheme:

\section{Development projects / implementation projects}

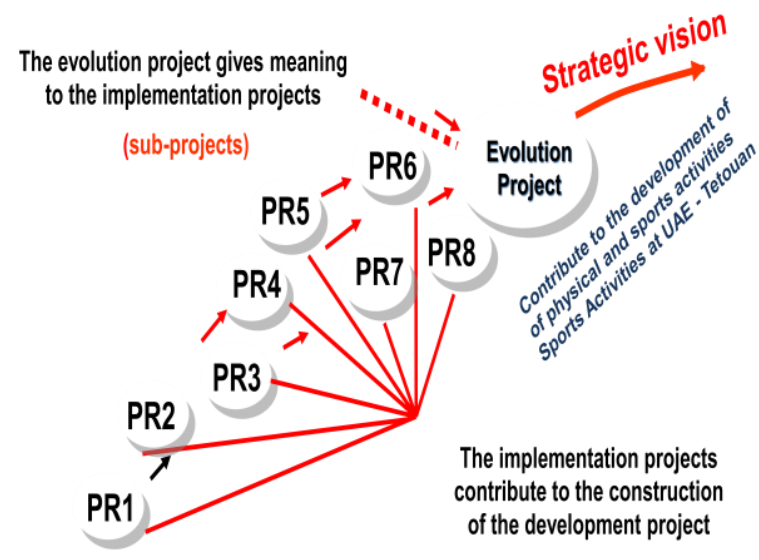

Figure 10. Projet d'évolution / projets de réalisation. 
Furthermore, it is a question for school leaders not to reduce their sports mission around a humanistic, social and recreational orientation. The restructuring of the sport/EPS system should focus equally on the concept of sports performance, winning medals, organizing sports events and improving the competitiveness of the UAE teams.

The model shown below also applies to the other institutions involved in this development project. It represents the way in which the UAE-SDP (Strategic Development Project) will be operationally broken down into sub-projects (implementation projects) in a limited and well-defined context. The project management team of the HSE (a UAE institution) sets up one or more implementation projects (this project may concern mass sport or elite sport, the construction or rehabilitation of the institution's infrastructure, a partnership or sponsorship agreement, the creation of a STAPS course or the development of a training programme, etc.). Depending on the goal, it rigorously establishes the current situation within the HSE and formulates realistic and clear objectives, with qualitative and quantitative indicators, thus reflecting the desired situation. Then, action plans will be drawn up to achieve the desired result. Finally, the budget to be mobilised must be evaluated and potential sources of funding must be indicated. It will then be time to move on to the next action or another one.

The consolidation of the operationalization sheets for each university results in the UAE SDP

The operationalisation sheet represents the basic tool for the operationalisation of the UAE SDP (e.g. HSE implementation project)

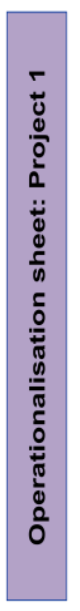

Project management team

Implementation project HSE

Current situation

Target situation

Quantitative Qualitative

targets

targets

Implementation budget

Implementation action plans

UAE SDP $n$

The consolidation of the operationalisation sheets of each academic institution gives rise to the UAE SDP

Figure 11. Operationalisation of the UAE SDP (e.g. HSE implementation project) b. Establishing a culture of coordination and communication

Furthermore, the total lack of coordination between the Social, Cultural and Sports Affairs Unit (CASCS) and the sport department at the UAE on the one hand, and between the sport department at the UAE and the department in charge of sport management in each university on the other hand, poses the serious problem of governance of sport in the university. This results in disparate actions and initiatives without a consistent scope. It subsequently becomes urgent to clarify the missions of each structure and to ensure mechanisms of cooperation and coordination between these entities so that they become more functional. A real coordination between them, but also with the multiple actors of university sport: Royal Moroccan Federation of University Sport, University Clubs, Regional Leagues, University Services of Physical and Sports Activities, student associations, Professional Licence MAS etc., would allow to rationalise and give a sense to the actions developed. A meaning that could be given by the President of the University...

The members of the Commission in charge of social, cultural and sports affairs (UAE) as well as the people in charge of the sports service at the UAE as well as in the different establishments of the University should be selected on the basis of their motivations (professors and others), their experience in the field or by considering their competences in the management and the animation of social, cultural or sports activities.

In the same vein, the difficulties of communication with superiors and those in charge of sports services at different levels should be overcome. The University, like the EU, should also activate and update the "SPORT" section of their official websites.

We also suggest the creation of a special website dedicated to sports activity at the league level, based at the UAE.

The optimisation of communication will attract more students to university sports activities, which may improve their level of commitment and motivation towards sports services in general.

In the same sense, and in order to overcome the problem of communication, an awareness-raising policy will be designed, centred on the creation of a newspaper for the University, the specific objective of which will be to invite the actors (at all levels: administrators, supervisors, coaches, licence-holders, etc.) to become impregnated with the values conveyed by university sport. This will promote a better knowledge of the sector and will surely motivate the actors to identify with it.

In our view, communication is a key prerequisite for the success of the SDP-EU. Communication must prepare 
the ground, primarily internally, to ensure an effective launch of the project.

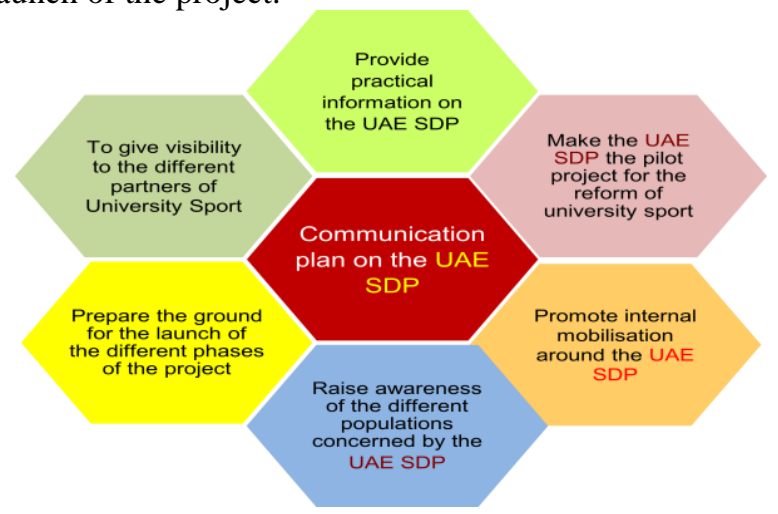

Figure 12. SDP-UAE Communication Plan.

c. Continuous evaluation of actions taken

In another register, improving the governance of PE and sports activities at the UAE must involve the continuous evaluation of this system as part of the university's teaching and training offer. The university and its institutions will proceed to the punctual evaluation of their actions by issuing reports, accounts and assessments that can be used for the purposes of verification and improvement. In concrete terms, toolkits adapted to each level of intervention of the actors involved should be set up. The contents of the SDP-EU monitoring toolbox, developed jointly, could take this form!

\section{The contents of the toolkit}

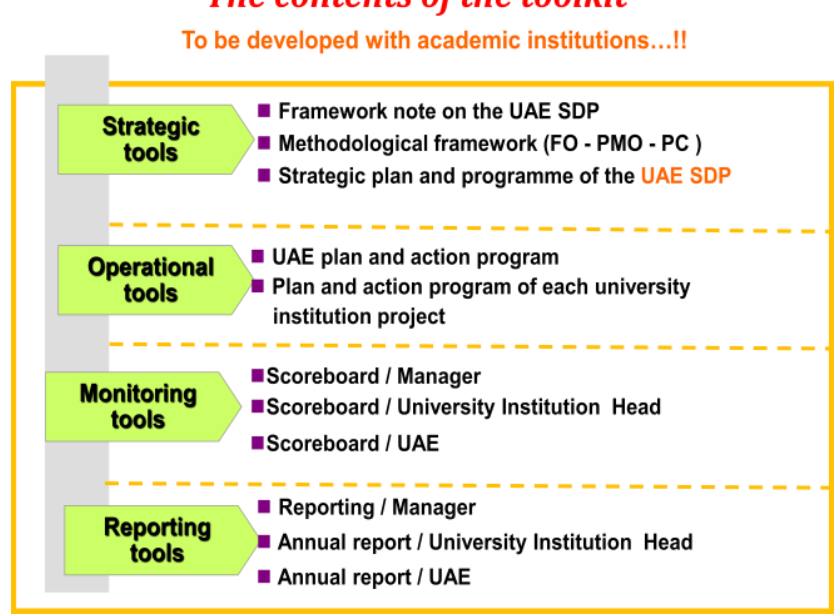

Figure 13. Contents of the UAE SDP Monitoring Toolkit.

The toolkit aims to achieve the following objectives:

- Support the project leaders in the formalisation of the operationalisation sheets ;

- Facilitate the implementation of the contractualisation ;
- Standardise the monitoring system of the SDPUI (University Institution).

d. To encourage and strengthen acts of partnership with its sporting, educational and civil environment.

Despite the dozens of cooperation agreements that the UAE has signed with organisations and universities in various countries: France, Spain, Tunisia, Egypt, Indonesia, Japan and the United States, it has practically no partnership agreement in the field of sport with any public or private sports organization. Faced with this situation, we are working towards opening up to the local, regional and national sporting, educational and civil environment.

The objectives are summarised as follows:

- Sharing of expertise between the UAE and public and private organisations, institutions and companies ;

- Pooling of financial, material and human resource ;

- Setting up innovative projects to support the sportivisation of university life within the UAE institutions ;

- Voluntary or philanthropic contributions (donations, legacies) compatible with the university's mission...

In concrete terms, we favour four types of partnership that can be granted by the UAE given the multiple elements of its environment:

1. Partnerships of a sporting nature: mainly aimed at Moroccan sports federations seeking to jointly:

- Expansion of the sports base ;

- Union of human, material and organisational capacities ;

- Establishing the relationship between school, university and civil sport ;

- Promotion of university sport and human development within the framework of sport ;

- Initiating extra-curricular programmes and activities with the aim of corporatising sportsmanship among students.

We will initially choose the following federations:

- Royal Moroccan Football Federation ;

- Royal Moroccan Athletics Federation;

- Royal Moroccan Basketball Federation.

We are also targeting the major sports clubs in the cities of the Tangier-Tetouan region.

2. Economic partnerships: mainly aiming at the same objectives as sports partnerships, they have a clear purpose: to find an official sponsor like "La centrale laitière" for school sports.

3. Partnerships in the social field: We look at: 
- $\quad$ The Red Crescent Association with the aim of sponsoring students' health, ensuring a new medical culture among students and their teachers ;

- Other associations that share similar ideas and development plans.

4. Institutional partnerships in the educational field:

- AREF Tangier-Tetouan-El Hoceima and its provincial branches ;

- Other Moroccan and foreign universities ;

- Royal Moroccan Federation of University Sport, Collectivités territoriales ;

- Royal Moroccan Federation of School Sport and the Directorate for the Promotion of School Sport ;

- Local authorities.

\section{Conclusion}

Practising sport is a constitutional right recognised to every Moroccan citizen. The university remains a privileged space for the practice of this right. As sport is generally absent from the development project of Abdelmalek Essaâdi-Tetouan University and its institutions, we have attempted to formalise a project for the development of the sport sector in the context of this Moroccan public university. Our proposal seeks to positivise the negative indicators and, at the same time, conceives the implementation of a rational and operational approach aiming at the restructuring of the university sport on more convincing managerial bases.

The perspectives identified are therefore centred on strengthening the means, raising the awareness of the actors and improving the organisation and management of physical and sports structures and activities.

Moreover, the effectiveness of the proposed solutions depends more on the willingness of those in charge (in particular the President and the heads of the various establishments covered by the UAE) to adopt the project and to provide the actors with the necessary resources.

The commitment and motivation of those involved in sport at the UAE, communication, consultation and dialogue between the different protagonists of university sport are therefore imperative for the development of the university sports community. The human factor is finally the guarantee of the success of this project.

Finally, it should be stressed that UAE sport is not totally isolated from the national university sport system. It is part of that system and should be influenced by it. Thus, trying to improve the UAE's sports offer becomes more or less dependent on what is happening further upstream; the Royal Moroccan Federation of University Sports, national federations, etc. Also, the regulations in force at the national level sometimes limit certain successful initiatives. The LMD system, combined with a semester system, a modular organization and continuous assessment, leaves students with less free time for physical exercise and sports and makes the programming of sustained sports activities problematic.

\section{REFERENCES}

[1] Harfouf S., (2021). Sport and Physical Education at Abdelmalek Essaâdi University: State of the Art. International Journal of Information Technology and Applied Sciences ISSN (2709-2208) Int. J. Inf. Tec. App. Sci. 3, No.2 (April-2021), https://doi.org/10.52502/ijitas.v3i2.40

[2] Royal letter from His Majesty King Mohamed VI to the National Sports Conferences, 2008.

[3] Ben Abdessalam M. \& Hasnaoui K., (2012). University sport in Morocco. Dissertation presented for the master's degree in management and engineering of sports organizations, Med V-Souissi University, Rabat.

[4] Harfouf S., (2008). School and university sport in Morocco from 1960 to the present day. Course that we gave to the students of the 3rd year of the cycle of preparation to the aggregation of Moroccan EPS. Academic year: 2007-2008. Unpublished document.

[5] National Strategy for the Development of Sport, Ministry of Youth and Sport, 2008.

[6] We refer especially to the initiative of the National School of Commerce and Management (ENCG)-Tangier, which is part of the UAE and which formulated a reform plan in 2010 but which has not been completed. (We interviewed the director of the ENCG in 2012 as part of a study on the subject).

[7] The UAE's sports actors also agree, and at the same time, on the need to reflect on the problems of sport and physical education at the grassroots level (at school and university). Ad hoc and sectoral initiatives have been tried without producing the desired effect.

[8] Harfouf, S., (2021). op. cit.

[9] Garel G., (2011). L'évolution des modèles de management de projet. In: Le management de projet. Paris, La Découverte, «Repères», p. 29-47. URL: https://www.cairn.info/le-management-de-projet9782707169891-page-29.htm

[10] Charue-Duboc, F., et Midler, C. Engineering activity and the concurrent project model. In Sociologie du travail [On line], Vol. $44-\mathrm{n}^{\circ} 3 \mid$ July-September 2002, online since 17 October 2002, accessed 26 april 2021. URL: http://journals.openedition.org/sdt/33858 ; DOI: https://doi.org/10.4000/sdt.33858

[11] Takeuchi, H. and Nonaka, I., (1986). The New Product Development Game. Harvard Business Review, 64, 137 . 146.

[12] Harfouf, S., (2021). op. cit.

[13] Oubahammou, L. Project Management, (2007). Presentation on the occasion of the implementation of a project on the implementation of Physical and Sports Education in the primary cycle. We participated in this project as a member of the team evaluating the physical and physiological capacities of primary school pupils in the Greater Casablanca area.

[14] L'idée de création d'un centre d'incubation des Activités Sportives Universitaire n'est pas originale. Elle a déjà été proposée par dans le cadre d'un projet de développement du Sport à l'Université Hassan2-Casablanca.

[15] We start from the assumption, supported by data, that the resources that can be made available for this project if it is adopted are not enormous. Therefore, we focus more on the rational use of existing resources. 
[16] By virtue of Dahir $n^{\circ} 1-10-150$ of 13 Ramadan 1431 (24 August 2010) promulgating the law 30-09 relating to physical education and sports \& Decree $\mathrm{n}^{\circ} 2-10-628$ of $13 \mathrm{di}$ hija 1432 (4 November 2011) of 29 April 1994 taken for the application of the law $\mathrm{n}^{\circ} 30-09$ relating to physical education and sports. Physical and sports education is not compulsory in higher education. However, in each institution of higher education, a university sports association must be created in order to allow students to express themselves physically and to participate in the various competitions organized by the FRMSU, by the regional leagues, by the sports association of the institution or others (clubs...).

[17] The act of partnership and cooperation signed between AREF and the UAE in 2007 should facilitate this operation. 